\title{
APPLICATION DE LA METHODE DE SOMMATION DE FAISCEAUX GAUSSIENS AU CALCUL DU CHAMP SONORE DIFFRACTÉ PAR UN ÉCRAN EN ATMOSPHÈRE INHOMOGÈNE
}

\author{
Y. GABILLET, H. SCHROEDER, G. DAIGLE* et A. L'ESPERANCE* \\ C.S.T.B., 24 rue Joseph Fourier, F-38400 Saint Martin d'Hères, France \\ *NRC, Institute for Microstructural Sciences, Ottawa KIA OR6, Canada
}

\begin{abstract}
:
The method of summation of Gaussian beams is an effective method for the computation of scalar wave fields (and vector flelds) in high frequency approximation $[1,2,3]$. This method is free of certain raytracing artifacts (infinitely high energy at caustics, perfect shadows) with the same computing cost. But, this method is not convenient for problems where there are rays tangent to the boundaries or where the boundaries have angular points. However, associated with the concept of the Geometrical Theory of Diffraction, it is possible to build a solution for the fields diffracted by an angular point. At this point, the incident field induces a cylindrical wave which can be decomposed in a set of gaussian beams. This method has been applied to the case of diffraction of the screen on the ground in the presence of downward refraction. The theory is compared with controlled measurements made indoors above a curved surface with a screen
\end{abstract}

\section{I - Introduction}

La méthode de sommation de faisceaux gaussiens est une méthode asymptotique permettant de résoudre certains problèmes de propagation en milieu inhomogène $[1,2.3]$. Elle a été appliquée au calcul des effets du vent et des gradients de température sur la propagation acoustique sur sol plan [4].La méthode de sommation de falsceaux gaussiens est très bien adaptée aux problèmes ne faisant pas intervenir des rayons tangents aux frontières et où les frontières elles-mêmes n'ont pas de discontinuité. Ceci est dû au fait que quand un faisceau gaussien est tangent à la frontière ou quand il intercepte une discontinuité, le champ réfléchı (diffracté) ne peut pas être décrit par un faisceau gaussien isolé. Cependant compte tenu des potentialités de la méthode pour résoudre certains problèmes de propagation acoustique extérieure (effet de gradients de vent et température), la solution a êtét modiffée pour intégrer ces effets de diffraction

\section{La sommation de faisceaux gaussiens}

Le principe de la méthode de sommation de faisceaux gaussiens consiste à éffectuer un tracé de rayons, puis à résoudre l'équation de Helmholtz dans un système de coordonnées centré sur le rayon en utisant l'approximation parabolique.

La solution parabolique au voisinage d'un rayon s'écrit :

$\tilde{u}(s, n, \omega)=\sqrt{\frac{c(0) q(0)}{c(s) q(s)}} \exp \left[-j \omega\left(\tau(s)+\frac{1}{2} M(s) n^{2}\right)\right]$

où: $s$ est la distance le long du rayon depuis la source, $n$ est la distance sur la perpendiculaire au rayon, $c(s)$ est la vitesse locale du son, $\tau(s)$ est le temps de propagation le long du rayon et $M(s)=p(s) / q(s)$ est donné par les fonctions: $p(s)=\varepsilon p_{1}(s)+p_{2}(s)$ et $q(s)=\varepsilon q_{1}(s)+q_{2}(s)$ avec $\varepsilon=\varepsilon_{1}+j \varepsilon_{2}$ $\varepsilon_{2} \geq 0$, où $\varepsilon$ est le paramètre complexe définissant le faisceau gaussien et $\left[p_{i}, q_{i}, i=1,2\right]$ sont les deux solutions indépendantes réelles du système d'équations différentielles linéalres du premier ordre obtenues à partir de l'équation de Helmholtz en appliquant l'approximation parabolique [1].La condition $\varepsilon_{2}>0$, garantit que l'énergle est confinée dans une région proche du rayon central. 
Pour considérer un point source dans un milleu 2-D plutôt qu'une ligne source [2], 1l suffit de multiplier l'expression (1) par le facteur d'amplitude tranerse au point récepteur $R$ :

$$
A_{y}(R)=\left(\int_{0}^{R} c(s) d s\right)^{-1 / 2}
$$

La dernjère étape de la méthode de sommation de falsceaux gaussiens consiste à superposer tous les faisceaux gaussiens passant dans le voisinage du point récepteur.

Désignons par $\alpha$ l'angle de tir d'un rayon par rapport à un axe du repère associé à la source. L'amplitude $\tilde{u}(s, n, \omega)$ du faisceau gaussien associé se calcule à partir de l'expression (1).

Le champ total en un point récepteur $\mathrm{R}$ s'écrit :

$U(R, \omega)=\int \Phi(\alpha, \omega) \tilde{u}(s, n, \omega) d \alpha$

La fonction de pondération $\Phi(\alpha, \omega)$ est déterminée en milieu homogène en comparant cette solution et la solution analytique pour une ligne source.

L'évaluation numérique de l'expression (2) consiste à la discrétiser en choisissant un ensemble de rayons $\alpha_{i}$, telle que l'expression (2) est remplacée par :

$U(R, \omega)=\sum_{i=0}^{N} \Phi\left(\alpha_{1}, \omega\right) \tilde{u}\left(s_{1}, n_{1}, \omega\right) \Delta \alpha_{i}$

où $\Delta \alpha_{1}$ est choisi suffisamment petit pour interpoler correctement l'intégrale (2).

\section{3 - Decomposition du champ diffracté par un ecran en une somme de faisceaux gaussiens.}

Dans le cas du problème de diffraction par une discontinuitè de la frontlère (on se limite aux discontinultés de type géométriques : écran-dièdre), deux démarches sont envisageables :

1) Rechercher la solution du problème de diffraction pour un faisceau isolé et intégrer cette solution dans la sommation.

2) Calculer le champ total incident sur l'arête diffractante et modéliser la diffraction en substituant à l'arête diffractante une source cylindrique décomposable en faisceaux gaussiens.

La première mêthode a été êtudiée par l'êquipe de FELSEN en électromagnétisme et sismologie [5]. Elle fait intervenir la notion de rayon complexe. Cette technique permet de simuler la diffraction par un écran plan en espace libre, mais est difficile à mettre en oeuvre dans le cas d'un écran ou d'un obstacle quelconque posé sur le sol dans un milieu dans lequel la vitesse du son varie avec l'altitude.

Contrairement à cette mêthode, où l'on considère la diffraction élémentaire par chaque faisceau gaussien pour calculer la diffraction totale, dans la 2ème méthode le champ diffracté est décomposé en une somme de faisceaux gaussiens. Si l'on reprend le principe du calcul du champ diffracté tel qu'll est défini dans la Théorte Géométrique de la diffraction [6], ce champ s'exprime par la relation sutvante :

$P\left(\rho, \rho^{\prime}\right)=P_{i}\left(0, \rho^{\prime}\right) D\left(\phi . \phi^{\prime}\right) G\left(\rho, \rho^{\prime}\right) e^{-j k \rho}$

Où $\rho . \rho^{\prime}, \phi, \phi^{\prime}$, sont définis à la figure 1 .

Dans cette relation. $P_{i}\left(0, \rho^{\prime}\right)$ désigne le champ incident sur l'arête de l'écran, $D\left(\phi_{.} \phi^{\prime}\right)$ désigne un coefficient de diffraction, $G\left(\rho, \rho^{\prime}\right)$ désigne un coefficient de divergence et jkp un terme de phase.

Si on se limite, dans un premier temps, au problème à deux dimensions, cette représentation du champ diffracté revient à considérer une source linėique sur l'arête de l'écran quil rayonne dans tout l'espace.Pour appliquer le formalisme des faisceaux gaussiens, il faut donc trouver la décomposition en faisceaux gaussiens de cette source de diffraction. Le champ incident $P_{i}\left(0, \rho^{\prime}\right)$ peut être calculé de manière globale comme la sommation des faisceaux gaussiens arrivant dans le voisinage de l'arête de diffraction.Pour trouver la décomposition d'une ligne source diffractante en une somme de faisceaux gaussiens, il suffit d'identifier la valeur asymptotique de l'expression (2) en milieu homogène avec la solution analytique (4) en vue d'en dédulre l'expression de $\Phi(\alpha, \omega)$ : 
$\Phi(\alpha, \omega)=-\frac{P_{i}\left(0, \rho^{\prime}\right)}{2} \frac{\exp \left(-j \frac{\pi}{2}\right)}{2 \pi} \sqrt{\frac{\rho^{\prime}}{\rho+\rho^{\prime}}}\left[\frac{1}{\cos \left(\frac{\phi-\phi^{\prime}}{2}\right)}+\frac{1}{\cos \left(\frac{\phi+\phi^{\prime}}{2}\right)}\right]$

Dans cette relation le champ incident $\mathrm{Pi}\left(0, \rho^{\prime}\right)$ est calculé comme la somme des faisceaux gaussiens arrivant dans le voisinage du récepteur.

Cette dernière relation, introduite dans l'expression du champ total (2) permet de calculer le champ diffracté par un écran droit comme une somme de falsceaux gaussiens issus de l'arête.

Il n'existe pas dans la littérature de données ou mesures du champ diffracté par un écran dans un milieu où la vitesse du son varie linéairement avec l'altitude. En utilisant l'analogie entre un terrain plat avec rayons courbés en atmosphère stratifiée, et une surface courbée au-dessus de laquelle il n'y a pas de réfraction, il est possible de réaliser sur maquette de telles mesures. Utilisant cette analogie, nous avons comparé les résultats de mesure derrière un écran droit de hauteur $15 \mathrm{~cm}$ posé sur une surface courbée qui est une section transversale d'un cylindre de rayon $20 \mathrm{~m}$, avec les résultats du calcul sur sol plan mais dont la célérité varie avec l'altitude suivant la loi: $c=c_{0}(1+0.05 z)$.

Les comparaisons entre résultats expêrimentaux et résultats théoriques de l'atténuation par rapport au champ libre dans la gamme de fréquences $1 \mathrm{kHz}-10 \mathrm{kHz}$ pour 2 récepteurs placés à $2 \mathrm{~m}$ derrière l'écran et respectivement à $10 \mathrm{~cm}$ et à $15 \mathrm{~cm}$ de hauteur sont données aux figures 2 et 3 . L'accord entre calcul et mesure est très bon. Pour cette configuration, bien que le récepteur soit atteint par les rayons directs et réfléchis, done dans la zone "éclairée", l'effet de diffraction est très important comme le montrent les rêsultats de mesures et de calculs pour la même configuration mais en absence d'écran (figure 2 - a , figure 3 - a).

Dans les configurations réelles, le gradient de célérité n'est en général pas linéaire et, en présence de vent, le profil de célérité est fortement perturbé dans le voisinage de l'écran. Néanmoins, cette nouvelle approche permet d'avoir une metlleur idée de l'efficacité réelle des écrans acoustiques en bordure de voie routière et plus généralement d'améliorer les outils de prévision de la propagation du bruit en extérieur.

\section{4 - Conclusions}

Une méthode mixte, incluant le concept de sommation de faisceaux gaussiens et la Théorie Géométrique de la Diffraction, a été développée pour traiter le cas de propagation acoustique en milieu inhomogène en présence d'obstacle. Le phênomène de diffraction par une arète est modélisé par le rayonnement d'une source équivalente placée au point de diffraction . Cette source est décomposée en une somme de faisceaux gaussiens dont la pondération est obtenue à partir de la théorle géométrique de la diffraction. Une première expérlence réalisée en modèle réduit dans le cas d'un gradient linéaire a permis de montrer la valıdité de cette méthode.

Cette étude a bénéficié du soutien de la Direction des Recherches Etudes Techniques .

\section{References}

[1] V. CERVENY, M.M. POPOV, and I. PSENCIK 1982, Geophys. J.R. Astron, Soc. 70, 109128,

"Computation of wave fields in homogeneous media. Gaussian beam approach".

[2] V. CERVENY, I. PSENCIK 1984, Geophy J.R. Astron. Soc, 78, 65 - 91,"Gaussian beams in elastic

2 - D laterally varying layered structures"

[3] M. WEBER 1988, Geophysical Journal 1988, 92, 9 - 24, "Computation of body-wave seismograms

in absorbing 2-D media using the gaussian beam method : comparison with exact methods".

[4] Y. GABILLET, M. ROSEN1989 - Inter-Noise 89,Newport Beach,CA,USA, "Computation of the wind effect on outdoor sound propagation by use of the gaussian beam tracing method".

[5] L.B. FELSEN, 1984, Geophys. J.R. Astr. Soc 79, 77-88, "Geometrical theory of diffraction, evanescent waves, complex rays and gaussian beams"

[6] R.G. KOUYOUMJIAN and P.H. PATHAK, 1974, Proc I.E.E.E. 62, 1448 - 1481, "A uniform geometrical theory of diffraction for an edge in a perfectly conducting surface". 


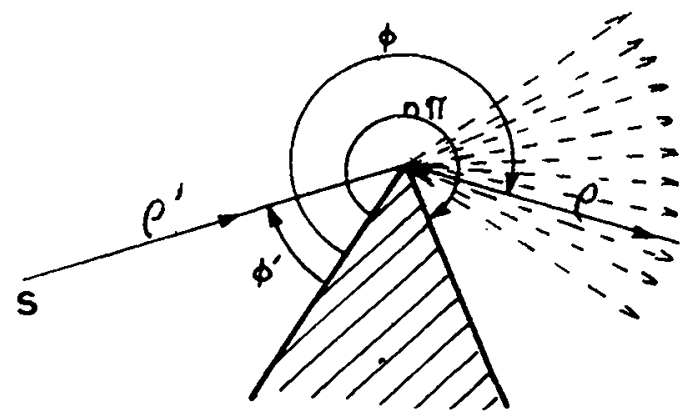

Figure 1 : Définition des paramètres utilisés dans le calcul du coefficient de diffraction

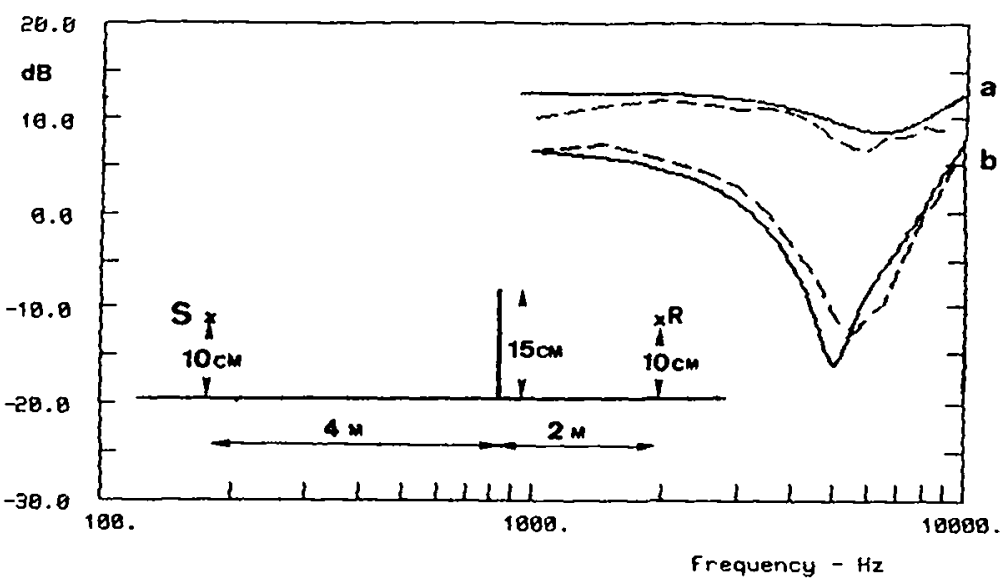

Figure 2: Attênuations du nłveau de pression par rapport au ntveau en champ libre mesurée(- - - ) et calculée(- $\rightarrow$ en fonction de la fréquence, sur sol réfléchissant, pour un récepteur à $10 \mathrm{~cm}$ au dessus du sol. La vitesse du son est définie par $c=340(1+0.05 \mathrm{z})$.

$$
\begin{aligned}
& \text { a - configuration sans écran } \\
& \text { b - configuration avec écran }
\end{aligned}
$$

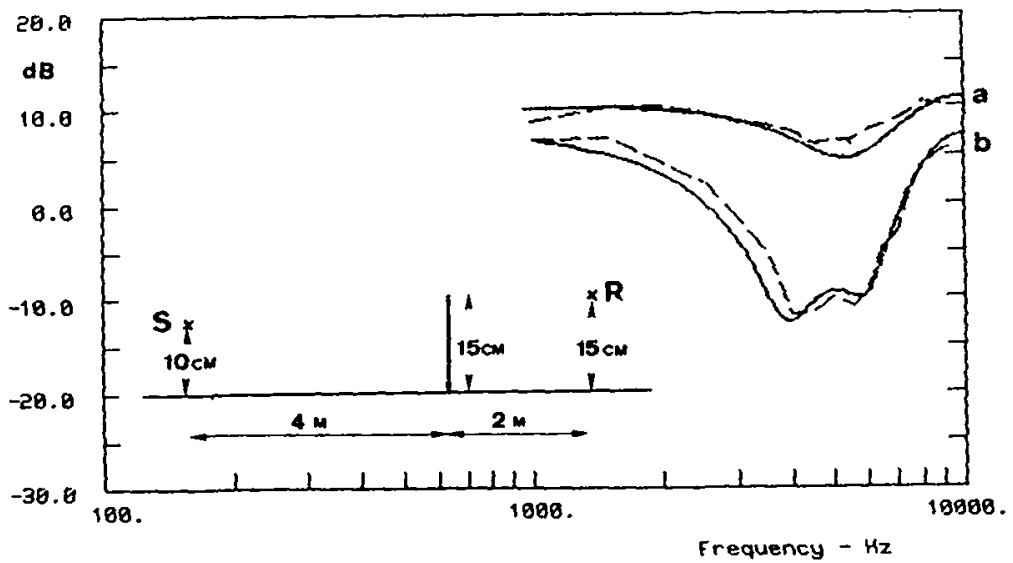

Figure 3 : Configuration identique à la figure 2 pour un récepteur à $15 \mathrm{~cm}$ au dessus du sol. 